livraisons

d'Histoire

de l'Architecture

\section{Livraisons de l'histoire de l'architecture}

39 | 2020

Maquettes d'architecture

\title{
L'histoire de l'architecture en un coup d'œil : quelques notes sur les maquettes et la construction de discours historiques entre Paris et Londres à l'époque des révolutions (1790-1830)
}

The history of architecture at a glance: some notes on architectural models and the construction of the historical speeches between Paris and London at the time of the revolutions (1790-1830)

Architekturgeschichte auf einen Blick. Anmerkungen zu Architekturmodellen und zum historischen Diskurs zwischen Paris und London zur Revolutionszeit (1790-1830)

\section{Adrián Almoguera}

\section{OpenEdition}

Journals

Édition électronique

URL : https://journals.openedition.org//ha/1585

DOI : 10.4000/lha.1585

ISSN : 1960-5994

Éditeur

Association Livraisons d'histoire de l'architecture - LHA

Édition imprimée

Date de publication : 15 juin 2020

Pagination : $77-88$

ISSN : 1627-4970

Référence électronique

Adrián Almoguera, «L'histoire de l'architecture en un coup d'œil : quelques notes sur les maquettes et la construction de discours historiques entre Paris et Londres à l'époque des révolutions

(1790-1830) », Livraisons de l'histoire de l'architecture [En ligne], 39 | 2020, mis en ligne le 24 janvier 2021, consulté le 21 septembre 2021. URL : http://journals.openedition.org//ha/1585 ; DOI : https:// doi.org/10.4000/lha.1585 


\section{L'HISTOIRE DE L'ARCHITECTURE EN UN COUP D'EIL : QUELQUES NOTES SUR LES MAQUETTES ET LA CONSTRUCTION DE DISCOURS HISTORIQUES ENTRE PARIS ET LONDRES À L'ÉPOQUE DES RÉVOLUTIONS}

(1790-1830)

\section{Des origines en papier : imaginer, publier et comparer l'histoire de l'architecture}

À la fin du XVIII ${ }^{\mathrm{e}}$ siècle, époque marquée par la découverte, la classification et la diffusion écrite et visuelle de la connaissance universelle, s'impose en Europe une vision cyclique de l'histoire qui s'applique bien évidemment à celle de l'art. Depuis les publications de Winckelmann, l'idée supposée d'une origine, apogée et décadence de l'art dans l'Antiquité est reprise à l'époque où Edward Gibbon, Giovanni Battista Piranesi, Quatremère de Quincy ou Seroux d'Agincourt essaient d'appliquer cette vision à leurs disciplines d'étude respectives. En ce qui concerne l'art de bâtir, bien avant Winckelmann, l'intérêt fut celui de pouvoir jouir d'une vision globale de l'histoire de l'architecture " en un coup d'œil ». Sur ce sujet, la première grande référence théorique et visuelle à avoir eu un impact général sur la pensée architecturale européenne est, bien sûr, l'Entwurff einer Historischen Architektur publié en 1721 par Johann B. Fischer von Erlach (ill. 1). L'abondante littérature de voyage produite et richement illustrée dans la seconde moitié du siècle n'a fait qu'accélérer un processus d'expansion des horizons théoriques et visuels qui a nécessité une méthode de classification et de comparaison scientifique ${ }^{1}$. C'est ainsi qu'est né le parallèle d'architecture, compris comme « la représentation de plusieurs édifices ou formes architecturales sur une même planche gravée ", grâce auquel on pouvait établir des comparaisons historiques sur l'évolution d'un type architectural à travers les différents peuples, parfois y rajoutant des exemples contemporains (ill. 2) ${ }^{2}$. Ce genre de publication, qui arrive à son sommet avec le Recueil et parallèle de Jean-Nicolas-Louis Durand, complété théoriquement en 1809 par l'Essai sur l'histoire générale de l'architecture publié par Jacques-Guillaume Legrand, est le fruit d'une évolution dans la culture visuelle des Lumières qui passe par le Parallèle publié par Meissonier vers 1750 et, notamment, par la célèbre planche comparative

1. Nous trouvons une liste de ces Voyages pittoresques dans Werner Szambien, Jean-Nicolas-Louis Durand, 1760-1834: de l'imitation à la norme, Paris, Picard, 1984, p. 170.

2. Cité dans Werner Szambien, Jean-Nicolas-Louis Durand..., op. cit., p. 28. Voir également JeanPhilippe Garric, Recueils d'Italie, les modèles italiens dans les livres d'architecture français, Bruxelles, Mardaga, 2004, p. 112-119. 


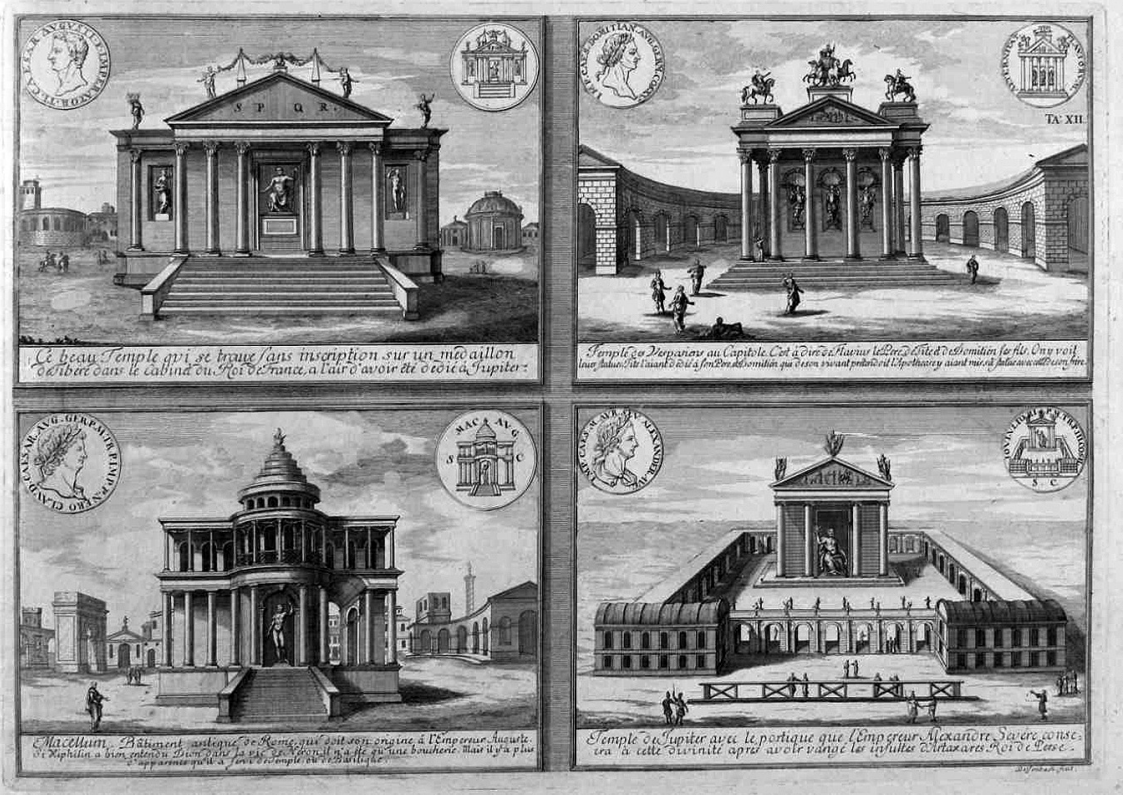

Ill. 1 : Johann B. Fischer von Erlach, Entwurf einer Hisorischen Architectur, Vienne, 1721, pl. XII.

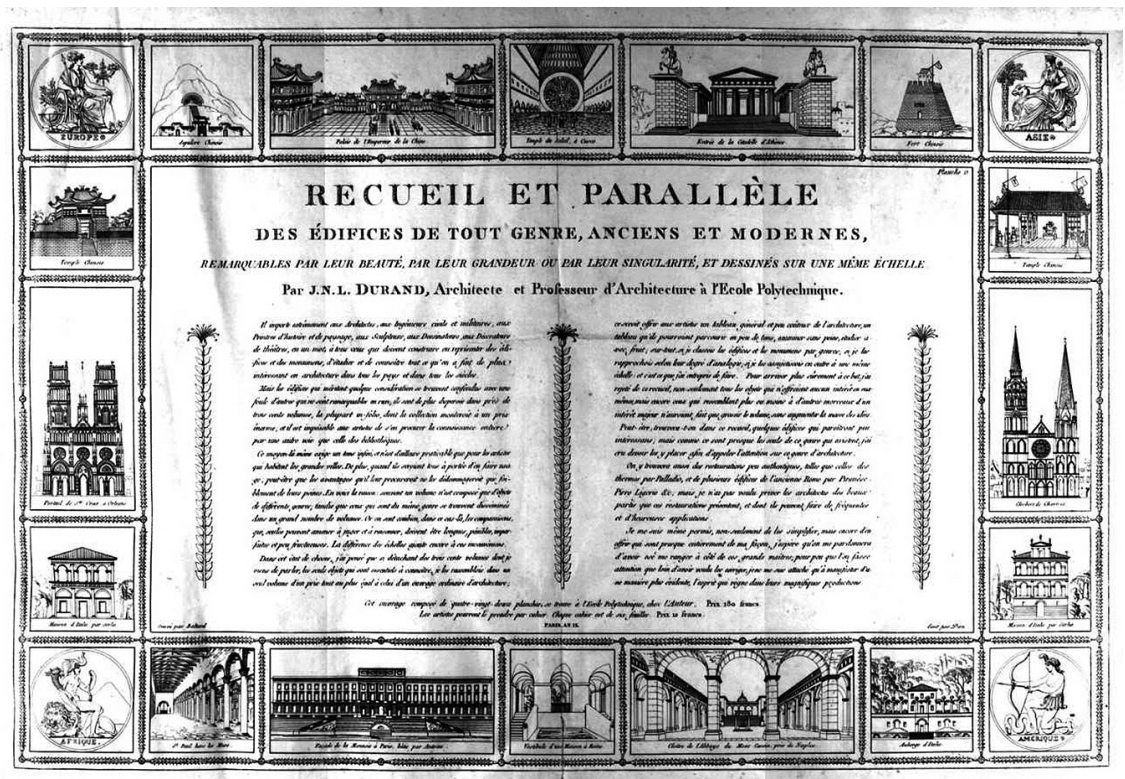

Ill. 2 : Frontispice du Recueil et parallèle des édifices de tout genre, gravé par Baltard, avec texte écrit par Dien, an IX. 
sur les temples égyptiens, phéniciens, grecs, romains et chrétiens publiée par Julien David Leroy dans l'édition de 1770 de ses Ruines des plus beaux monuments de la $G_{\text {rèce }}{ }^{3}$. Ces parallèles, où des types architecturaux séparés dans le temps et dans l'espace géographique étaient comparés sur une même échelle, témoignent d'une nouvelle manière de comprendre l'histoire de l'architecture, pas toujours forcement dans un sens évolutif. Ainsi, une nouvelle démarche comparative sur la multiplicité de formes et de modèles dans l'histoire globale de l'architecture se consolide à l'époque de l'effondrement du système vitruvien. À partir de ce cadre théorique et visuel, des initiatives pour l'aménagement d'espaces d'exposition comparative de cette nouvelle vision de l'histoire de l'architecture fleurissent. Dans ce contexte, aux dessins et gravures typiquement utilisés pour ce faire, se rajoute l'usage des maquettes utilisées dans la constitution de galeries d'architecture comparée, ancienne et moderne, capables d'offrir "en un coup d'œil" une connaissance naguère incontournable pour stimuler l'imagination des architectes et combler les nouveaux besoins de formes et usages demandés par l'architecture nouvelle de l'époque des révolutions en Europe.

\section{La galerie de maquettes en tant que récit historique dans le Paris de la Révolution}

À l'aube de la Révolution, à Paris, les deux principales collections semi-publiques de maquettes d'architecture se trouvaient à l'Académie royale d'architecture et à l'École des Ponts et chaussées, où elles étaient l'un des piliers pédagogiques dans la formation des jeunes architectes et ingénieurs. Or, dans le cadre de la révision de l'histoire de l'art globale orchestrée autour de la constitution du Museum révolutionnaire entre 1790 et 1794, les maquettes d'architecture sont placées au cœur des initiatives pour aménager au Louvre un espace d'exposition de l'histoire de l'architecture universelle suivant une vision préconisée par la production littéraire et graphique citée auparavant.

À cette époque, la politique de séquestres des biens appartenant aux aristocrates exilés a favorisé l'incorporation au patrimoine de l'État de nombreuses maquettes d'architecture qui devaient se mettre au service du programme pédagogique de la République. Ainsi, au début des années 1790, on saisit les célèbres collections du comte de Choiseul-Gouffier, grand voyageur et diplomate, et du comte d'Orsay, entre autres ${ }^{4}$. Dans son ouvrage sur la question, Werner Szambien a remarqué le rôle joué par ces deux collections de maquettes, riches en modèles des monuments grecs et romains, dans les projets de constitution d'une section d'architecture au Louvre. Pour ce faire, la commission temporaire des arts et le conservatoire des arts étaient chargés de "chercher tout ce qui peut donner une idée des divers modes

3. Nous renvoyons vers Christopher Drew Amstrong, Julien David Leroy and the Making of Architectural History, Londres, Routledge, 2012, p. 156-182.

4. Voir Werner Szambien, Il Museo di architettura, Bologne, CLUEB, 1996, p. 52-59. 
de construire des peuples anciens » dans le but de constituer des espaces d'étude à travers " toutes les maquettes d'architecture qui peuvent être utiles à l'histoire ou aux progrès de cet art ${ }^{5}$.

L'incorporation au patrimoine de la nation de ces richesses artistiques, jadis appartenant exclusivement à la sphère du dilettantisme aristocratique, était un geste politique fort souvent souligné par les idéologues de cette politique. Ceux-ci se vantaient de ce trésor qui "dans ses mains [celles des émigrés] servait seulement à solliciter la vanité ou à orner les salons $"{ }^{6}$. Le passage du cabinet privé à la galerie publique de maquettes d'architecture devait servir à démocratiser la connaissance de la culture architecturale des peuples du passé, notamment la Grèce et l'Empire Romain, devenus par ailleurs des modèles culturels, artistiques et moraux de la République, et plus tard de l'Empire français. Ainsi, ces maquettes d'architecture, qui étaient en fin de compte des objets artistiques de luxe, devaient servir à manifester publiquement la conquête de l'histoire et du passé culturel au profit du discours du progrès historique de la nation française. Cependant, très tôt, les projets pour aménager une section d'architecture au Louvre perdent leur importance dans les grandes initiatives concernant le musée. Ainsi, lorsqu'en 1793, Jean-Baptiste-Pierre Lebrun rédige ses Quelques idées sur la disposition [...] du musée, il accorde seulement une section pour l'exposition de maquettes, qui devaient uniquement concerner les monuments égyptiens exposés sous la forme de "petits modèles tracés sur des échelles exactes de leurs immenses pyramides, leurs sarcophages, leurs momies, en un mot, tout ce qui peut nous éclairer sur ces peuples, les plus anciens qui soient connus " ${ }^{7}$.

Ces tentatives d'aménagement d'une galerie de maquettes d'architecture au Louvre visaient fondamentalement à créer un discours sur le passé architectural des civilisations qui se trouvaient à l'origine d'une évolution historique dans laquelle la France contemporaine voulait jouer un rôle dominant. C'est par ailleurs dans ce contexte que s'est produit l'événement clé utilisé comme emblème de cette politique culturelle pré-impériale de réécriture historique : l'entrée au Louvre du cortège cérémoniel des trésors artistiques d'Italie en 1798. Cependant, pour compléter ce discours sur le passé architectural des peuples annexé à la gloire nationale, à Paris, on commence à vanter la production architecturale contemporaine, qu'il fallait préserver pour l'histoire comme on le faisait avec les monuments anciens. Pour ce faire, on n'a pas hésité à demander la création de maquettes servant à illustrer un nouveau chapitre de l'histoire de l'architecture portant sur la grandeur de l'art de bâtir de la France révolutionnaire.

5. Procès-verbaux du Conservatoire des arts datés du 12 janvier et 11 février 1795 à propos de la collection Choiseul-Gouffier, qu'on veut "transférer dans le Musée ", cités dans Yveline CantarelBesson, La Naissance du Musée du Louvre, Paris, RMN, 1981, t. I, p. 130.

6. Rapport fait à la Convention Nationale au nom du Comité d'instruction publique, 28 frimaire an III, cité par G. J. Guillaume (dir.), Procès-verbaux du Comité d'instruction publique de la Convention Nationale, t. III, p. 171.

7. Jean-Baptiste-Pierre Lebrun, Quelques idées sur la disposition, l'arrangement et la décoration du Muséum national, Paris, Didot jeune, s.d. [1794-1795], p. 13. 
Ainsi, dans le but de constituer une galerie des meilleures productions de l'architecture contemporaine, lors des célèbres concours de l'an II, le jury chargé de récompenser les projets vainqueurs décide de commander des reproductions dans le but de "former, ensemble à ceux qu'on peut rajouter annuellement, une collection de maquettes" pour illustrer ainsi les progrès de la nouvelle architecture publique issue des idéaux révolutionnaires ${ }^{8}$. L'idée chère à la pensée académique des Lumières, et très développée pendant la Révolution et le Premier Empire est de créer des collections témoignant de la production architecturale contemporaine. Il faut constituer des espaces d'exposition d'architecture comparée permettant de parcourir visuellement, d'un coup d'œil, le perfectionnement de l'art de bâtir à cette l'époque9. L'objectif principal étant de lier la culture visuelle des modèles du passé, dessinés, moulés ou construits en miniature, avec le nouveau répertoire de la production contemporaine ${ }^{10}$. À travers ces maquettes, les pouvoirs publics, sous la Révolution, voulaient affirmer que la nouveauté contemporaine avait dépassé les modèles des anciens à travers l'invention de nouveaux types d'édifices marqués par une iconographie architecturale renouvelée ${ }^{11}$.

Or, dans ce contexte parisien de propositions pour la création d'espaces d'exposition d'une histoire de l'architecture à travers les maquettes, les deux seules expériences vraiment abouties sont celle de Louis-François Cassas, et celle entreprise conjointement par les architectes Jacques-Guillaume Legrand et Jacques Molinos ${ }^{12}$. Ces derniers ont acheté au début des années 1790 une maison au n 6 de la rue Saint-Florentin à Paris où ils ont aménagé une galerie de maquettes d'architecture antique complétées par des dessins et des gravures. La particularité de cette galerie était son intérêt pour l'évolution historique de l'architecture d'ordre dorique, objet d'étude fondamental de la nouvelle architecture au tournant du XIX ${ }^{\mathrm{e}}$ siècle. Celle-ci avait trouvé dans le prestige du primitivisme et la solidité de l'architecture dorique des peuples anciens une alternative valable au système vitruvien. Ainsi, même l'édifice qui contenait la collection avait été conçu suivant un programme d'architecture

8. Werner Szambien, Il Museo di architettura..., op. cit., p. 49. L'auteur signale l'existence de quelques maquettes élaborées d'après cette initiative, repérées au collège des Quatre-Nations en 1806. Nous renvoyons également vers Werner Szambien, Les Projets de l'an II, concours d'architecture de la période révolutionnaire, Paris, ENSBA, 1994.

9. Werner Szambien, Il Museo di architettura..., op. cit., p. 129-137 retrace des tentatives d'aménagement de "modèles d'architecture " dans les différents projets d'établissement académiques proposés pendant le Premier Empire à Paris.

10. L'échec de la création d'un espace public pour l'exposition de l'architecture contemporaine fut en partie comblé par des publications comme la Collection des prix de l'Académie, publiée à partir de 1787, les Annales du musée, publiées par Charles-Paul Landon depuis 1801, et le Recueil d'architecture nouvelle, publié par Athanase Détournelle à partir de 1805.

11. J. A. Leith, The Idea of Art as Propaganda in France 1750-1799, Toronto, 1965, p. 178 analyse les 150 projets architecturaux présentés aux salons entre 1789 et 1799 , dont presque un tiers étaient des projets révolutionnaires.

12. Sur l'association de ces deux architectes voir A. C. Quatremère de Quincy, Recueil de notices historiques, Paris, Adrien Le Clere et $C^{\text {ie }}$, 1834, p. 75-91 et Werner Szambien, Il museo di architettura..., op. cit., p. 71-77. 
caractéristique sur les façades, ornées de pilastres et de colonnes doriques sans base, encore visibles aujourd'hui. Par ailleurs, le parcours historique à travers les chefsd'œuvre de l'architecture dorique présenté dans cette galerie, se poursuivait dans la cour de l'édifice, où les architectes avaient aménagé une reproduction d'une colonnade du Parthénon, ainsi que des colonnes «des propylées d'Athènes, du temple de Thésée, du portique d'Auguste à Athènes, du grand temple de Paestum [...] " ${ }^{13}$. Lors de son ouverture, en 1800, la galerie d'architecture dorique de Legrand et Molinos est devenue le premier espace d'exposition semi-public consacré à l'histoire de l'architecture de France.

Six ans après le début de cette expérience, l'architecte et grand voyageur LouisFrançois Cassas inaugurait en 1806 au 8, rue de Seine un véritable musée d'architecture comparée composé de maquettes, gravures et dessins proposant une histoire visuelle de l'art de bâtir dans l'Antiquité (ill. 3) ${ }^{14}$. Cet ensemble, exposé aussi dans une galerie, se composait de maquettes d'architecture "cyclopéenne, celtique et druidique ", égyptienne, indienne, persane, grecque, étrusque, romaine et " du bas empire ". Au-delà de sa diffusion par la presse rattachée à l'intelligentsia parisienne, comme l'Athenaeum, le contenu de cette galerie d'architecture antique comparée a fait l'objet d'une description concise par Jacques-Guillaume Legrand ${ }^{15}$. Les maquettes de Cassas ont été conçues avec une harmonie dans l'échelle qui fait penser aux parallèles imprimés à l'époque, notamment au Recueil et parallèle de Durand, publication qui était censée condenser pour ses lecteurs une sorte de connaissance universelle en un coup d'œil. L'intention de Cassas était surtout de stimuler l'imagination des artistes de son temps grâce au véritable voyage à travers le temps et les formes que proposait cette collection à titre de modèles pour la nouvelle architecture historiciste explorée tout au long du XIX ${ }^{\mathrm{e}}$ siècle. Suite à un rapport favorable signé par Durand, Vaudoyer et Dufourny en 1808, l'État entame une procédure d'achat de la collection Cassas, finalement aboutie en 1813, ce qui marque le début d'une lente dispersion de ces maquettes dans les fonds des institutions culturelles et d'enseignement ${ }^{16}$.

\section{L'histoire de l'architecture à la maison: à propos des maquettes chez John Soane}

C'est en effet à peu près à l'époque des premiers travaux pour la galerie d'ordre dorique rue Saint-Florentin, que John Soane a commencé à constituer sa collection artistique, surtout enrichie de fragments d'architecture (anciens ou sous la forme

13. Legrand et Molinos, "Lettre aux rédacteurs ", Journal des arts, des sciences et de la littérature, 10 mars 1800, p. 1-2.

14. Sur Cassas, voir notamment les deux articles publiés par H. Boucher, "Louis-François Cassas ", Gazette des Beaux-Arts, 1926, t. II, p. 21-53 et 209-230.

15. Jacques-Guillaume Legrand, Collection des chefs-d'euvre de l'architecture des différens peuples : Exécutés en modèles, sous la direction de L.-F. Cassas..., Paris, Leblanc, 1806. Cassas lui-même publie une Notice des chefs-d'ceuvre de l'architecture des différens peuples représentés dans son musée.

16. Voir à ce sujet Jannic Durand, "Une collection oubliée: les maquettes anciennes du Musée des Antiquités nationales ", Antiquités nationales, n 14-15, 1982-1983, surtout p. 122-135. 


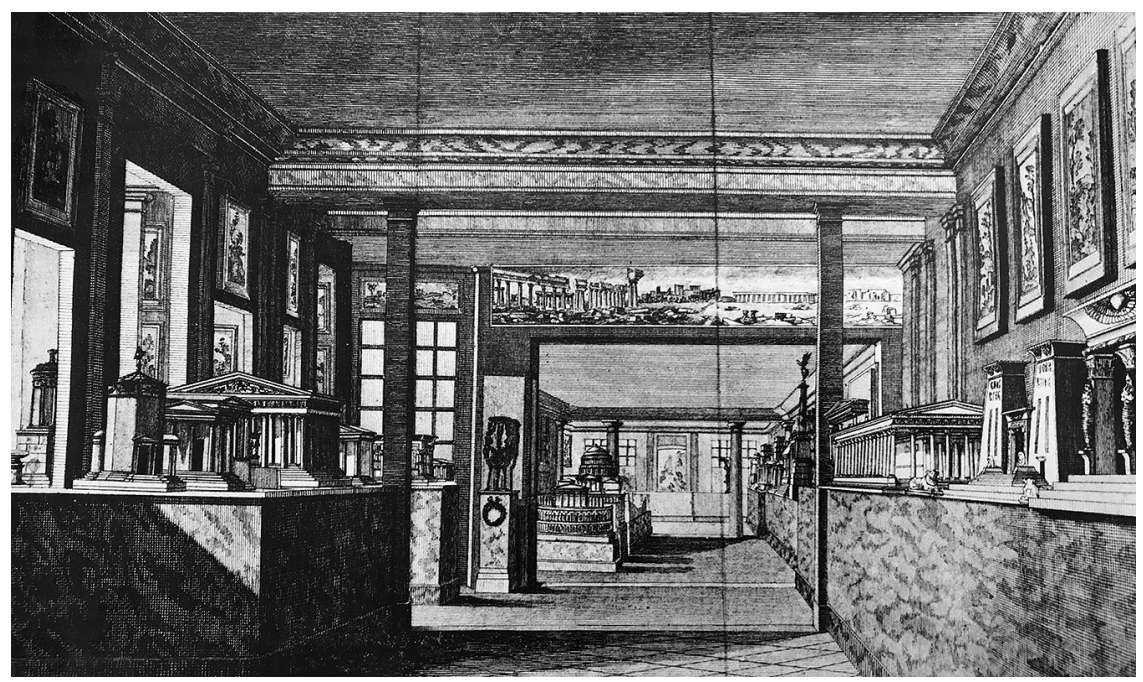

Ill. 3 : Vue générale de la Galerie des chefs-d'euvre de l'architecture des differens peuples de François Cassas, gravure publiée par Pierre-Louis Baltard dans la revue Athenaeum, 1806.

de moulages), d'une vaste collection de peintures, et d'une collection incontournable de dessins, gravures et maquettes d'architecture représentant l'histoire de cet $\operatorname{art}^{17}$. La particularité de cette collection de modèles architecturaux était sans doute la riche présence de maquettes des projets conçus par l'architecte, c'est-à-dire, d'une micro-histoire de l'architecture contemporaine produite par Soane et disposée en paragone avec les modèles anciens qu'il avait thésaurisés chez lui. L'intention de l'architecte était clairement de marquer un continuum visuel et théorique entre sa production et celle des anciens, en s'inscrivant ainsi dans une sorte de lignée historique dont il se présentait comme l'héritier et le rénovateur ${ }^{18}$. Pour Soane, les maquettes d'architecture étaient fondamentales d'un point de vue pédagogique, constructif, historique et même créatif. L'architecte a exposé son avis sur la question dans l'une de ses célèbres conférences à la Royal Academy, pour lesquelles Soane s'appuyait toujours sur une vaste production de dessins, mais aussi de maquettes ${ }^{19}$. Du fait de son grand intérêt pour la question des origines et de l'évolution de l'art de bâtir, Soane a souvent encouragé une démarche comparative entre les modèles

17. Dans sa recension de l'ouvrage de Werner Szambien parue dans le Burlington Magazine, $\mathrm{n}^{\circ} 131$, 1989, p. 433-434, Barry Bergdoll signale la nécessité de mettre en rapport les expériences parisiennes avec les collections académiques italiennes et les collections privées de certains architectes dont, notamment, celle de John Soane que nous souhaitons, en suivant cette piste, relier au contexte parisien.

18. Sur cette collection, voir John Wilton-Ely, "The architectural models of Sir John Soane : a Catalogue ", Architectural History, vol. 12, 1969, p. 5-38.

19. La XII ${ }^{\mathrm{e}}$ conférence est reproduite dans David Watkin, Sir John Soane : Enlightenment Thought and the Royal Academy Lectures, New York, Cambridge University Press, 1996, p. 659-663. 


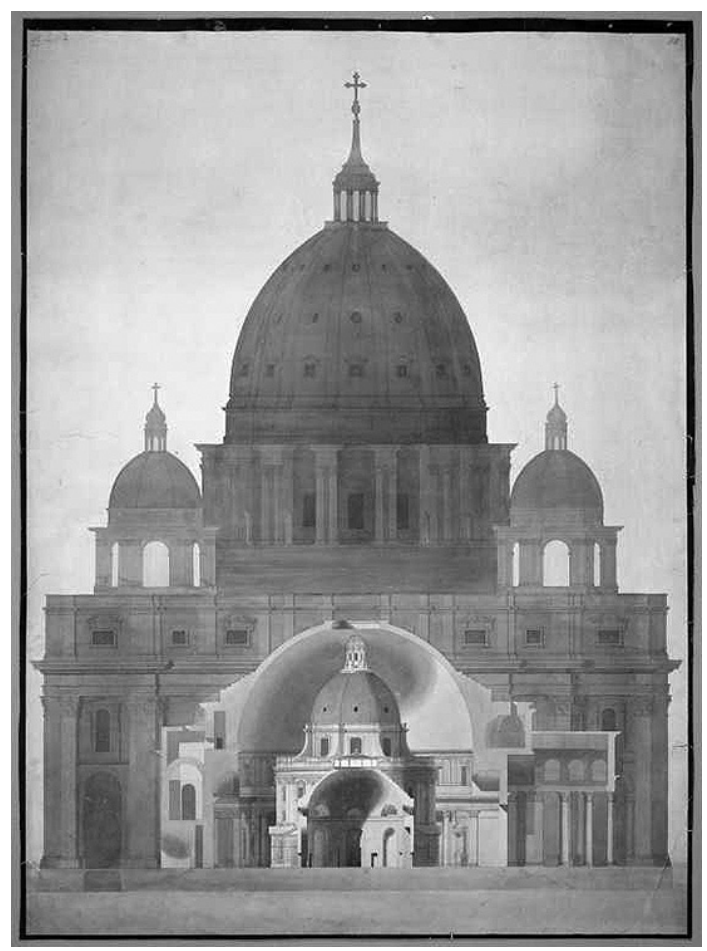

Ill. 4 : Cabinet de John Soane, Étude comparative entre Saint-Pierre de Rome et les sections du Panthéon, la Radcliffe Library à Oxford, et la rotonde de la banque d'Angleterre, dessin au crayon, encre de chine et lavis de couleurs, c. 1810, $78 \times 37$ cm. (C) Londres, Trustees of Sir John Soane Museum.

architecturaux du passé et du présent, qu'il faisait dessiner sur une même feuille avec la même échelle permettant ainsi, comme pour les maquettes, d'analyser l'évolution historique d'un certain type architectural, y compris à travers ses propres projets, en un coup d'œil (ill. 4$)^{20}$.

L'objectif de Soane, notamment en ce qui concerne l'usage des maquettes dans la pédagogie architecturale, pratique alors en désuétude dans son pays, était de produire "des impressions et des sensations de la plus grande hauteur, allant au-delà du pouvoir de la description... des sensations qu'on peut seulement dépasser par la contemplation directe des édifices eux-mêmes ${ }^{21}$. Cette idée était très proche du projet pédagogique de la galerie d'architecture comparée de Cassas. Elle était également inspirée de ces tableaux comparatifs qui parlent aux yeux, selon Legrand, qui vantait les initiatives, comme celle de Durand, pour « représenter réunis dans un

20. Cet intérêt historique est analysé à travers les sources théoriques étudiées par Soane (Fischer Von Erlach, Piranèse, Julien David Leroy, J.-N.-L. Durand...) dans David Watkin, Sir John Soane, Enlightenment..., op. cit., p. 98-183.

21. Ibid., p. 561. 
même cadre, et souvent sur une même échelle, l'immense variété des formes de l'architecture $" 22$.

L'objectif d'offrir une histoire comparée de l'architecture à travers ses collections remontait aux origines de la carrière de Soane, qui affirmait que «depuis ma nomination pour l'enseignement, j'ai commencé à disposer les livres, moulages et maquettes pour que les étudiants puissent bénéficier d'un accès facile " à ces sources ${ }^{23}$. En effet, contrairement à ce qu'on a pu affirmer sur ce sujet, Soane prend en considération cette problématique lorsqu'il dresse des projets d'aménagement pour ses deux principales demeures, toutes les deux conçues originairement comme une sorte de maisonacadémie-musée ${ }^{24}$. Que ce soit dans ses projets pour le Pitzhanger Manor, sa maison de campagne jusqu'en 1810, ou surtout dans sa célèbre demeure londonienne au 13 Lincoln's Inn Fields, l'architecte songe à l'aménagement de galeries d'exposition de dessins et maquettes illustrant une histoire de l'architecture allant de l'Antiquité à son propre travail. Dans cette optique, en 1810, l'architecte pense même à aménager dans sa maison londonienne deux galeries superposées de cent pieds de longueur contenant, des dessins et des gravures pour la première, des maquettes et des moulages d'édifices anciens et modernes pour la deuxième ${ }^{25}$. Ainsi, même si ces galeries n'ont pas été finalement construites, la maison de Soane était dès le début conçue comme une sorte de "musée éducatif semi public pour des étudiants en architecture ${ }^{26}$. Les maquettes d'architecture parsemaient les espaces du refuge piranésien de Soane, mais c'est surtout à l'intérieur de la Model Room, richement décrite par l'architecte lui-même, qu'il a mis en place un discours historique sur la continuité entre son œuvre et les grands monuments du passé qui l'avaient inspiré (ill. 5) ${ }^{27}$. Ainsi, entourées d'un ensemble de dessins et de gravures d'architecture, les maquettes des temples de Paestum, des fouilles à Pompéi, du Panthéon de Rome et du Temple de Vénus à Baalbek, dialoguaient avec les projets de Soane pour la Three per Cent Reduce Office ou la New Board of Trade and Privy Council Offices à Whitehall. De ce fait, à la manière d'un clin d'œil qui résume ce projet d'autoinclusion dans une microhistoire de l'architecture ancienne et moderne à travers les maquettes, Soane propose la comparaison visuelle entre la maquette du temple

22. Prospectus de Legrand dans l'édition italienne du Recueil de Durand (1833), cité par Werner Szambien, J.-N.-L. Durand..., op. cit., p. 170. John Soane, qui possédait par ailleurs un exemplaire de la Collection des chefs-d'œuvre publié en 1806 par Legrand, séjourna à Paris pendant trois semaines au mois d'août 1814, lorsque les maquettes de Cassas étaient pour la plupart stockées à l'École des Beaux-Arts. L'architecte britannique a-t-il eu l'opportunité de voir ces maquettes à cette occasion ?

23. Dans David Watkin, Sir John Soane, Enlightenment..., op. cit., p. 256.

24. Nous renvoyons aux pages d'introduction de l'édition italienne de l'ouvrage de Werner Szambien, Il museo di architettura..., op. cit., p. IX-XIV et 3-7. L'auteur insiste sur le manque de projet pédagogique chez Soane, hypothèse largement déconstruite par la bibliographie sur le sujet.

25. Voir Susan G. Feinberg, "The genesis of Sir John Soane's Museum idea : 1801-1810 ", JSAH, vol. 43, 1984, p. 236.

26. Ibid., p. 236-237.

27. John Soane, Description of the house and museum on the north side of Lincoln's Inn Fields, Londres, Levey, Robson and Franklyn, 1835, p. 87. 


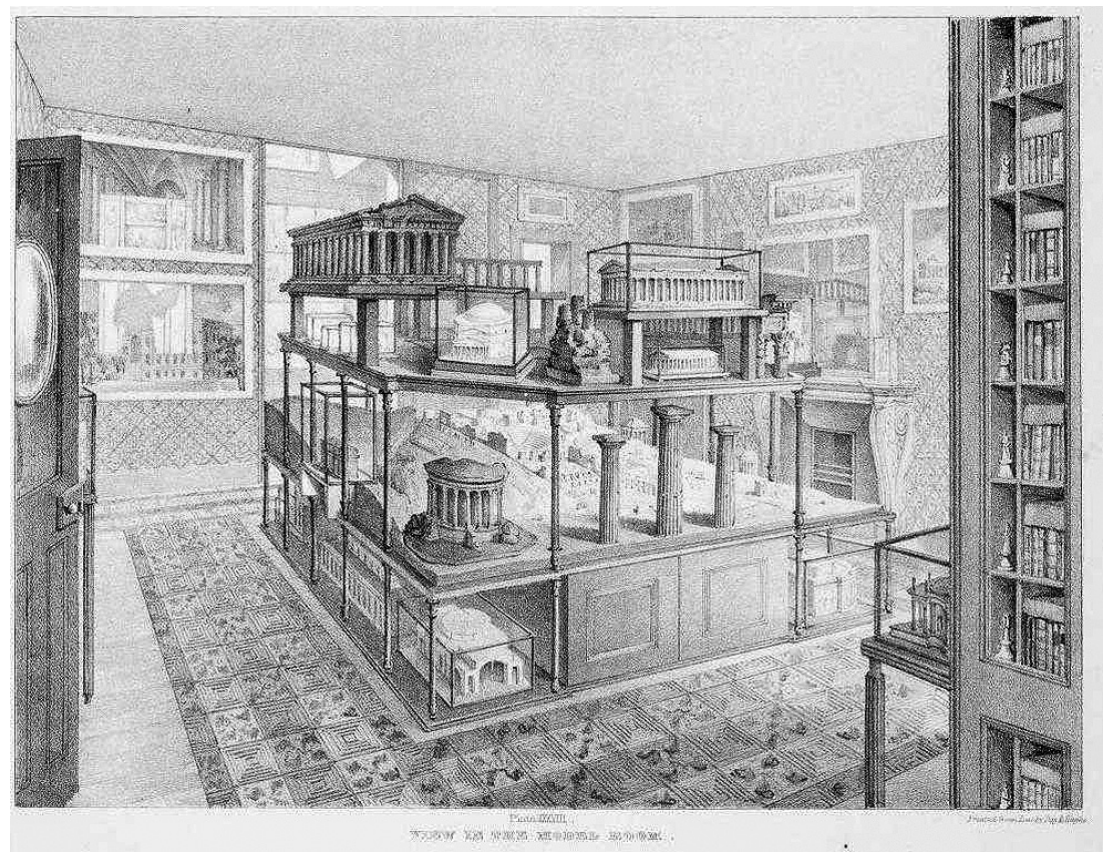

Ill. 5 : Vue de la model room au 13 Lincoln's Inn Fields, publiée dans John Soane, Description of the house and museum on the north side of Lincoln's Inn Fields, the residence of Sir John Soane, Londres, 1835, pl. XXXVIII.

de Vesta à Tivoli et le Tivoli Corner de la Banque d'Angleterre, l'un des projets qui a fait sa renommée internationale.

Par ailleurs, cette métaphore visuelle fut portée à son paroxysme à travers deux belles compositions de Joseph Michael Gandy, le dessinateur le plus prolifique du cabinet de Soane ${ }^{28}$. Dans celle que Gandy a exposée à la Royal Academy en 1818 (ill. 6), on peut voir un ensemble de plus de cent projets de Soane sous la forme de dessins et surtout de maquettes. Celles-ci entouraient l'auteur assis à son bureau, dessinant à côté de la maquette de la Banque d'Angleterre, doublement représentée en grand format, au sommet de la composition. Ce "rêve de l'architecte " imaginé par Gandy, résumait parfaitement la conception comparative de l'histoire architecturale qu'il avait appris entre les maquettes et les dessins de Soane, et à partir de laquelle il développe une production pendant les années 1830 regroupée sous la notion générale de Comparative Architecture sur les styles architecturaux du monde qu'il voulait publier dans une édition monumentale ${ }^{29}$. Par ailleurs, le format de

28. Voir à ce sujet William Palin, "J. M. Gandy's composite views for Sir John Soane », dans Lucien Steil (dir.), The architectural capriccio, memory, fantasy and invention, Farnham, Ashgate, 2014, p. 99-118.

29. Voir à ce sujet Brian Lukacher, Joseph Gandy, an Architectural Visionary in Georgian England, Londres, Thames and Hudson, 2006, p. 168-197. Le travail auprès de Soane est étudié aux pages 132-167. 


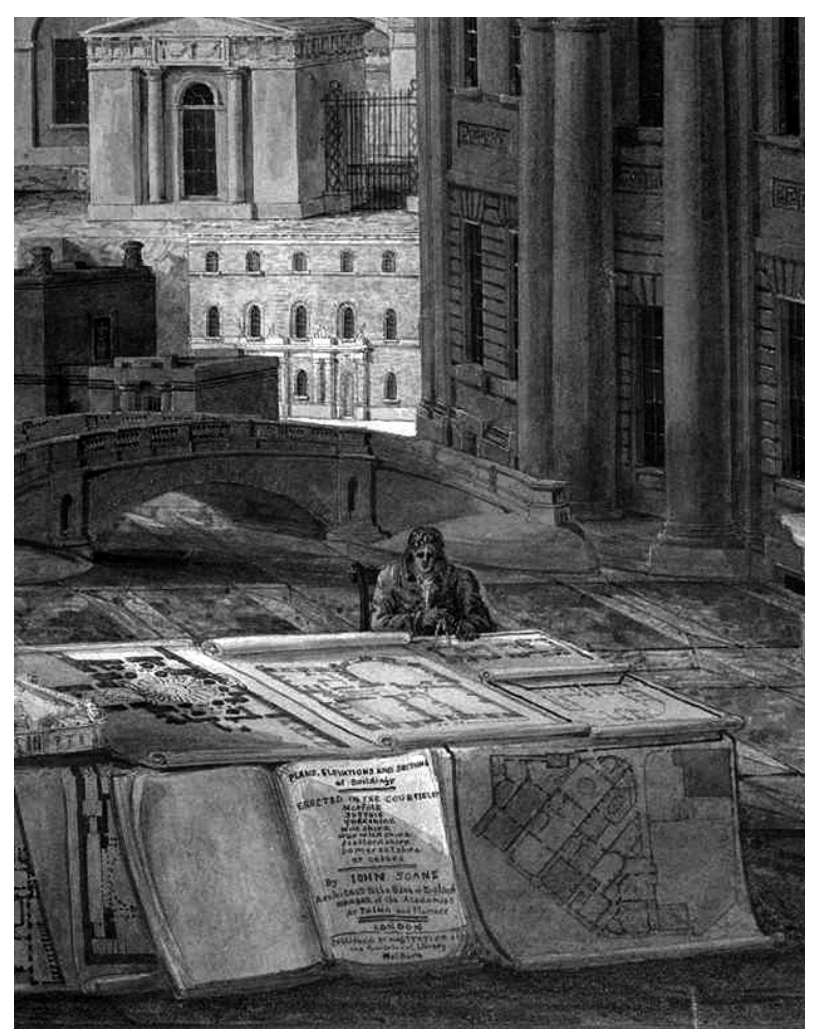

Ill. 6 : Joseph Michel Gandy, Composition architecturale avec des perspectives et des maquettes des projets faits par Sir John Soane entre 1780 et 1815, aquarelle, 1818. ( ) Londres, Trustees of Sir John Soane Museum.

cette sorte d'utopie visuelle qui encadre les micro-histoires de l'architecture conçues par Gandy, a débouché sur une série d'architectural visions dans le romantisme angloaméricain enrichies aussi bien par les compositions de Charles Robert Cockerell que par les peintures de Thomas Cole. Ainsi, la perspective historique développée par Soane à travers les collections de sa maison-musée londonienne a inauguré une manière de concevoir l'histoire visuelle de l'architecture qui a fait de cette expérience un moment unique dans l'évolution de la pensée architecturale européenne.

Héritiers d'une culture des Lumières partagée et construite entre la rêverie romaine et l'effervescence intellectuelle du tournant du XIX siècle, les acteurs principaux du phénomène que nous avons étudié dans cet article ont fondé leurs apports au discours historique général sur un point de départ commun marqué par la nouvelle culture livresque et théorique dégagée dans la seconde moitié du XVIII ${ }^{\mathrm{e}}$ siècle. Ainsi, de la planche à la maquette, du parallèle à la galerie d'architecture comparée, le besoin d'illustrer l'histoire, ou les histoires de l'architecture, a contribué à la construction de discours justement parallèles des deux côtés de la Manche. Dans 
ce contexte, la maquette d'architecture a été utilisée comme objet à la fois esthétique et pédagogique. Elle a été aussi un élément créateur d'une réflexion qui a contribué à la diffusion et à la multiplication de formes et d'inspirations dans l'architecture européenne du XIX ${ }^{\mathrm{e}}$ siècle, toujours partante pour l'expérimentation et la confrontation avec l'univers révélé par les recueils et les galeries de maquettes dans cette époque de révolutions politiques et culturelles.

Adrián Almoguera

Docteur en histoire de l'architecture, Sorbonne Université Membre de l'École française de Rome 